Using R331 renal carcinoma cells that express caspase-8 (FLICE)-like inhibitory protein (FLIP), which inhibits TRAIL-DR5-mediated apoptosis, the authors showed that DR5-mediated tumour-cell apoptosis was essential for trimAb-mediated tumour rejection. Interestingly, only a minor population (10\%) of DR5-susceptible tumour cells in the tumour burden was necessary for tumour rejection to occur in response to trim $A b$ therapy. This rejection required the activation of $\mathrm{CD}^{+} \mathrm{T}$ cells, indicating that both tumour-cell apoptosis through DR5 and tumour-specific CTL responses were required for tumour eradication by trimAb therapy.

So, combining agonistic monoclonal antibodies that activate T cells (CD40 and CD137) with an agonistic monoclonal antibody that induces tumour-cell apoptosis (DR5) is an effective tumourspecific immunotherapy in mice. This combination therapy might be a potential strategy for cancer immunotherapy in humans.

Olive Leavy

ORIGINAL RESEARCH PAPER Uno, T. et al. Eradication of established tumors in mice by a combination antibody-based therapy. Nature Med. 7 May 2006 (doi:10.1038/nm1405)

transferred to naive mice that were deficient for both RAG2 and the common cytokine receptor $\gamma$-chain. These mice, in addition to being B-celland T-cell-deficient, also completely lack NK cells. Subsequent challenge with DNFB resulted in a vigorous CHS response in these mice, confirming the ability of sensitized NK cells to mediate a hapten-specific memory response independently of B cells and T cells. These transferable hapten-specific NK memory cells were shown to localize in the donor's liver but not in the spleen.

These results describe an unexpected function for NK cells and indicate that a B-cell- and T-cell-independent adaptive immune response exists in mammals.

$\Rightarrow$ CYTOKINES

\title{
IL-25 expels worms
}

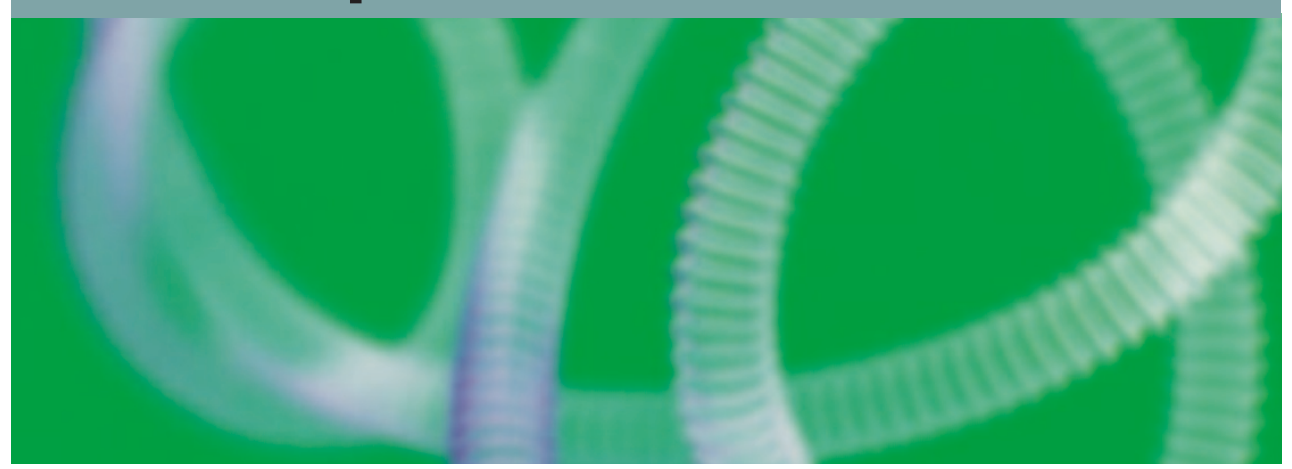

Two papers published recently in The Journal of Experimental Medicine provide new insight into the development of protective T helper 2 $\left(\mathrm{T}_{\mathrm{H}} 2\right)$-cell responses in vivo: they show that the cytokine interleukin-25 (IL-25) is an important regulator of $\mathrm{T}_{\mathrm{H}} 2$-cell responses.

It has been suggested that IL-25 (also known as IL-17E) is involved in the development of $\mathrm{T}_{\mathrm{H}} 2$-cell responses. Because immunity to infection with parasitic helminths is usually associated with $\mathrm{T}_{\mathrm{H}} 2$-cell responses, both groups studied the role of IL-25 in immunity and inflammation in vivo using mouse models of infection with such parasites.

Owyang et al. showed that, although AKR mice are normally susceptible to infection with Trichuris muris, treatment with recombinant IL-25 induced resistance to infection with this parasite. Resistance to infection was associated with a $\mathrm{T}_{\mathrm{H}} 2$-cell response, including increased production of IL-4, decreased production of interferon- $\gamma($ IFN $\gamma)$ and increased numbers of goblet cells in the caecum. Consistent with these observations, although wild-type C57BL/6 mice infected with $T$. muris mount an efficient $\mathrm{T}_{\mathrm{H}}$ 2-cell response and are resistant to infection, IL-25-deficient C57BL/ 6 mice infected with T. muris failed to mount a $\mathrm{T}_{\mathrm{H}} 2$-cell immune response and were unable to clear the worms from the gastrointestinal tract.

Similarly, Fallon et al. found that IL-25deficient mice were unable to clear the parasitic helminth Nippostrongylus brasiliensis as rapidly as wild-type mice. Delayed clearance of the parasite was associated with delayed induction of $\mathrm{T}_{\mathrm{H}} 2$-cell responses. For example, whereas in the presence of IL-25 large amounts of the $\mathrm{T}_{\mathrm{H}} 2$ cytokines IL-4, IL-5 and IL-13 were detected 5 days after infection, in the absence of IL-25 cytokines were not observed until 10 days after infection.

Owyang et al. found that blocking $\mathrm{T}_{\mathrm{H}} 1$-cell responses in IL-25-deficient mice infected with
T. muris reversed the decrease in production of $\mathrm{T}_{\mathrm{H}} 2$ cytokines and enabled the mice to clear the infection. Therefore, in addition to a role in the efficient induction of $\mathrm{T}_{\mathrm{H}} 2$-cell responses, IL-25 might have a role in the suppression of $\mathrm{T}_{\mathrm{H}} 1$-cell responses. Consistent with this anti-inflammatory role, chronically infected IL-25-deficient mice developed severe intestinal inflammation that was associated with high levels of pro-inflammatory cytokines such as IFN $\gamma$ and IL-17 in the caecum.

Using mice expressing a lacZ reporter construct in the Il25 locus, Owyang et al. were able to show that subsets of $\mathrm{CD} 4^{+}$and $\mathrm{CD} 8^{+} \mathrm{T}$ cells in the caecal patch (which is a lymphoid follicle closely associated with the mucosal surface) of mice resistant to infection with $T$. muris express IL-25. Furthermore, Fallon et al. showed that a population of non-B-cell, non-T-cell $\mathrm{KIT}^{+} \mathrm{Fc}_{\mathrm{CRI}}{ }^{-}$ cells responded to IL- 25 by producing large amounts of IL-4, IL-5 and IL-13. In resistant mice, the number of these cells in the mesenteric lymph nodes increased following infection with N. brasiliensis, whereas in IL-25-deficient mice the number of these cells remained static. The correlation between the number of these cells and the ability of the mice to clear $N$. brasiliensis led the authors to suggest that these cells are an initial source of $\mathrm{T}_{\mathrm{H}} 2$ cytokines following infection with $N$. brasiliensis.

These two studies clearly identify IL-25 as a regulator of $\mathrm{T}_{\mathrm{H}}$ 2-cell responses in vivo and, as Owyang et al. suggest, provide a new potential therapeutic target for the treatment of diseases associated with pathogenic $\mathrm{T}_{\mathrm{H}} 2$-cell responses.

Karen Honey

ORIGINAL RESEARCH PAPERS Owyang, A. M. et al. Interleukin 25 regulates type 2 cytokine-dependent immunity and limits chronic inflammation in the gastrointestinal tract.J. Exp. Med. 203, 843-849 (2006) | Fallon, P. G. Identification of an interleukin (IL)-25-dependent cell population that provides IL-4, IL-5, and IL-13 at the onset of helminth expulsion. J. Exp. Med. 203, 1105-1116 (2006) 